\title{
Effet du charbon des noyaux de fruits noirs (Canarium schweinfurthii) ou de rafles de maïs sur les performances de production des poulets de chair au Cameroun
}

\author{
J. R. KANA * , A. TEGUIA, B. M. MUNGFU et J. TCHOUMBOUE \\ Département des Productions Animales, Faculté d'Agronomie et des Sciences Agricoles, \\ Université de Dschang, BP : 188 Dschang, Cameroun \\ *Auteur correspondant, E-mail : kanajean@yahoo.fr; tel: +237 74893394
}

\section{RESUME}

Une étude a été menée sur l'effet des charbons des noyaux des fruits noirs (Canarium schweinfurthii Engl.) et de rafles de maïs (Zea mays L.) sur les performances de production et les caractéristiques de la carcasse des poulets de chair. A cet effet, 120 poussins mâles de souche Hybro ont été repartis dans 12 unités expérimentales de 10 poussins chacun et élevés sur litière. Chaque unité expérimentale a reçu au hasard l'une des 3 rations expérimentales dont un control $\left(\mathrm{R}_{0}\right)$, l'aliment additionné de $1 \%$ de charbon de fruits noirs $(\mathrm{Rc})$ ou de charbon de rafles de maïs ( $\mathrm{Rr}$ ) dans un dispositif complètement aléatoire à 3 traitements avec 4 répétitions chacun. Pendant toute la période de l'étude, l'incorporation du charbon dans l'aliment a significativement $(\mathrm{p}<0,05)$ réduit la consommation alimentaire et le gain de poids des animaux comparé aux animaux du lot témoin. A l'exception du foie, aucune différence $(p>0,05)$ n'a été notée entre les différents lots d'animaux pour ce qui est du rendement carcasse et du poids relatif des différents organes étudiés. Dans les conditions de cette étude, il a été conclu que l'inclusion des charbons de fruits noirs et de rafles de maïs n'a aucun effet bénéfique sur les performances de croissance et les caractéristiques de la carcasse chez les poulets de chair.

(C) 2009 International Formulae Group. All rights reserved.

Mots clés : Canarium schweinfurthii, Charbon, Cameroun, Poulets de chair, Zea mays

\section{INTRODUCTION}

Le secteur de la volaille est à la recherche des nouveaux additifs alimentaires qui stimulent la croissance et jouent un rôle important dans le contrôle de la population des microorganismes pathogènes tels que les salmonelles, les coliformes et favorisent le développement des microorganismes bénéfiques dans le tube digestif. La recherche des composés alternatifs aux antibiotiques, qui ont pourtant montré des effets bénéfiques, se justifie par le fait que leur utilisation régulière comme stimulateurs de croissance a développé des résistances chez les bactéries avec des conséquences potentielles sur la santé de l'homme (Jenser, 1998; Doyle, 2002; Wages, 2002; Al-harthi, 2006).

Les probiotiques faits à base des microbes vivants et qui jouent un rôle bénéfique dans l'équilibre de la flore intestinale de l'animal hôte (Woo et al., 2006; Gunal et al., 2006) et les acides organiques sont parmi les plus utilisés (Ahmad, 2006; Kyung Woo et al., 2006; Gunal et al., 2006; Soltan, 2008). Les microbes colonisent les parois intestinales et empêchent l'installation des microbes pathogènes, protégeant ainsi l'animal contre les maladies gastrointestinales (Ahmad, 2006). Les acides organiques quant à eux sont capables d'inhiber la croissance des microorganismes 
dans l'aliment et d'assurer l'équilibre de la flore bactérienne intestinale. Par ailleurs, en modifiant le $\mathrm{pH}$ intestinal, ces acides organiques améliorent aussi la solubilité des aliments, la digestion, l'absorption des nutriments et réduisent l'activité des enzymes des microbes indésirables dans l'intestin (Gunal et al,. 2006; Soltan, 2008). Toutefois, l'usage massif des microbes vivants comme probiotiques pourrait dans l'avenir poser des problèmes de santé publique. Par ailleurs, leur prix n'est pas à la porté de la bourse des éleveurs des pays pauvres. Il serait intéressant d'envisager d'autres substances naturelles à moindre coût, ne présentant pas de danger pour les animaux de la ferme et pour les consommateurs. Dans cet ordre d'idée, des études ont montré que les charbons ont la capacité de fixer les toxines (Ramos et al,. 1996), de favoriser le transit intestinal et jouent un rôle important sur le pansement gastrique (Cooney, 1980; Neuvonen et Olkkola, 1988). Ces qualités seraient de nature à améliorer la croissance des poulets.

C'est dans cette perspective que cette étude a été envisagée pour évaluer l'effet de deux charbons végétaux (Canarium schweinfurthii Engl. et de Zea mays L.) comme additif alimentaire sur les performances des poulets de chair.

\section{MATERIEL ET METHODES}

Site de l'essai et préparation des charbons

L'étude a été menée à la Ferme d'Application et de Recherche (F.A.R.) de l'Université de Dschang dans les hauts plateaux de l'Ouest-Cameroun. Cette localité est située à $05^{\circ} 26^{\prime}$ latitude Nord, $10^{\circ} 26^{\prime}$ longitude Est et à une altitude moyenne de $1420 \mathrm{~m}$. Les fruits noirs matures et les rafles de maïs collectés dans les exploitations paysannes autour de la ville de Dschang ont été calcinés et broyés dans un moulin pour en obtenir les farines de charbons.

\section{Matériel animal}

Un effectif de 120 poussins mâles d'un jour d'âge de souche Hybbro et d'un poids moyen de $37,5 \mathrm{~g}$ ont été répartis dans 12 unités expérimentales de 10 poussins chacun. Les poussins on été élevés sur litière profonde à une densité de $20 / \mathrm{m}^{2}$ jusqu'à l'âge de 3 semaines puis à une densité de $10 / \mathrm{m}^{2}$ jusqu'à 49 jours d'âge. Aussi bien en démarrage qu'en finition, 4 unités expérimentales ont été affectées au hasard à l'une des 3 rations expérimentales (Tableau 1) dont la ration de base qui servait de témoin $\left(\mathrm{R}_{0}\right)$, la ration de base additionnée de $1 \%$ de charbon des fruits noirs (Rc) ou de $1 \%$ de charbon de rafle de maïs ( $R r)$, dans un dispositif complètement aléatoire à 3 traitements avec 4 répétitions chacun.

Les poussins ont été vaccinés contre la maladie de Newcastle et la bronchite infectieuse le $8^{\text {ème }}$ jour avec un rappel le $23^{\text {ème }}$ jour et contre la maladie de Gumboro le $10^{\mathrm{e}}$ jour. Des vitamines et un anticoccidien étaient administrés dans l'eau de boisson trois jours de suite chaque semaine.

\section{Collecte et analyse statistique des données}

La croissance des poussins et la consommation alimentaire ont été évalués tous les 7 jours jusqu'à 49 jours d'âge. A cet âge, 2 poulets par unité expérimentale ont été sacrifiés pour l'évaluation de la carcasse. Le taux de la créatinine sérique a été déterminé par le test colorimétrique avec un kit commercial de la firme Bio Mérieux. La consommation alimentaire, le gain de poids moyen, l'indice de consommation, le rendement carcasse, le taux de la créatinine sérique et les proportions des organes internes ont été soumis à l'analyse de la variance suivant un dispositif expérimental complètement aléatoire et en cas de différence significative, les moyennes ont été séparées par le test de Duncan (Vilain, 1999).

\section{RESULTATS}

Le tableau 2 présente l'influence des rations sur les performances pondérales, la consommation alimentaire et l'indice de consommation (IC) cumulés des poulets durant les différentes phases de la période expérimentale. Lorsque l'on considère la phase de démarrage (1 à 21 jours), aucune différence significative $(\mathrm{P}>0,05)$ n'a été notée entre les animaux du lot témoin et les animaux ayant reçu les charbons végétaux pour tous les paramètres étudiés. Par contre, à l'exception de l'IC qui était statistiquement comparable $(\mathrm{P}>0,05)$ entre tous les lots sur toute la durée de l'essai, le gain de poids moyen et la consommation alimentaire moyenne cumulés du lot control étaient plus élevés $(\mathrm{P}<0,05)$ par rapport aux autres lots en phase de finition 
(22-49 j) et sur toute la période de l'étude (149 j). En examinant l'évolution des poids moyens des animaux des trois lots, on constate qu'elle est plus élevée $(\mathrm{P}<0,05)$ chez les animaux du lot témoin sur toute la période de l'étude par rapport aux lots qui ont reçu les charbons végétaux tel que l'illustre la figure 1 .

Il ressort du tableau 3 qu'à l'exception $\mathrm{du}$ foie, aucune différence significative
( $\mathrm{P}>0,05)$ n'a été notée entre les différents lots d'animaux pour ce qui est du poids relatif des différents organes étudiés et du rendement de la carcasse prête à cuir. Par contre, le taux de la créatinine était plus élevé $(\mathrm{P}<0,05)$ chez les animaux ayant reçu le charbon du noyau des fruits de Canarium par rapport aux animaux du lot témoin et ceux ayant reçu le charbon des rafles de maïs.

Tableau 1: Composition des rations expérimentales (\%).

\begin{tabular}{lcc}
\hline Ingrédients & Démarrage & Finition \\
\hline Maïs & 62 & 68 \\
Huile & 0,50 & $/$ \\
Tourteau de coton & 8 & 10 \\
Tourteau de soja & 20 & 11,50 \\
Farine de poisson & 3 & 4,20 \\
Farine d'os & 1,20 & 1 \\
Sulfate de fer & 0,30 & 0,30 \\
CMAV 5\% & 5 \\
Total & 5 & 100 \\
\hline Composition chimique calculée & 100 & \\
\hline PB $(\%)$ & 22,01 & 20,16 \\
EM (kcal kg) & 2969,50 & 3007,72 \\
Ca $(\%)$ & 1,02 & 1 \\
Phosphore disponible $(\%)$ & 0,58 & 0,57 \\
Lys $(\%)$ & 1,27 & 1,13 \\
Met $(\%)$ & 0,50 & 0,43 \\
\hline
\end{tabular}

${ }^{1}$ CMAV 5\%: $\mathrm{PB}=40 \%$, Lys $=3,3 \%$, Meth $=2,40 \%, \mathrm{Ca}=8 \%, \mathrm{P}=2,05 \%, \mathrm{EM}=2078 \mathrm{kcal} / \mathrm{kg}$.

Tableau 2: Effets du charbon de rafles de maïs et de fruits noirs sur quelques paramètres de production $(n=40)$.

\begin{tabular}{lccc}
\hline Paramètres & \multicolumn{1}{c}{ Ro } & Rc & Rr \\
\hline Poids initial & $37,5 \pm 0,40 \mathrm{a}$ & $37,6 \pm 1,54 \mathrm{a}$ & $37,25 \pm 1,19 \mathrm{a}$ \\
\hline Gain de poids $(\mathbf{g})$ & & & \\
\hline $1-21 \mathrm{j}$ & $550,42 \pm 38,12 \mathrm{a}$ & $515,20 \pm 34,14 \mathrm{a}$ & $514,47 \pm 32,47 \mathrm{a}$ \\
$22-49 \mathrm{j}$ & $1784,57 \pm 38,12 \mathrm{a}$ & $1636,70 \pm 42,36 \mathrm{~b}$ & $1576,89 \pm 76,91 \mathrm{~b}$ \\
$1-49 \mathrm{j}$ & $2284,13 \pm 227,84 \mathrm{a}$ & $2076,61 \pm 108,17 \mathrm{~b}$ & $2037,49 \pm 130,48 \mathrm{~b}$ \\
\hline Consommation alimentaire $(\mathbf{g})$ & & \\
\hline $1-21 \mathrm{j}$ & $941,78 \pm 66,36 \mathrm{a}$ & $892,80 \pm 27,49 \mathrm{a}$ & $872,93 \pm 74,38 \mathrm{a}$ \\
$22-49 \mathrm{j}$ & $4102,45 \pm 350,06 \mathrm{a}$ & $3916,15 \pm 183,49 \mathrm{~b}$ & $3820,53 \pm 233,65 \mathrm{~b}$ \\
$1-49 \mathrm{j}$ & $5044,23 \pm 444,91 \mathrm{a}$ & $4707,37 \pm 376,99 \mathrm{~b}$ & $4706,04 \pm 574,35 \mathrm{~b}$ \\
\hline Indice de consommation & & \\
\hline $1-21 \mathrm{j}$ & $1,58 \pm 0,34 \mathrm{a}$ & $1,64 \pm 0,46 \mathrm{a}$ & $1,60 \pm 0,27 \mathrm{a}$ \\
$22-49 \mathrm{j}$ & $2,42 \pm 0,43 \mathrm{a}$ & $2,49 \pm 0,46 \mathrm{a}$ & $2,44 \pm 0,20 \mathrm{a}$ \\
$1-49 \mathrm{j}$ & $2,06 \pm 0,57 \mathrm{a}$ & $2,11 \pm 0,54 \mathrm{a}$ & $2,13 \pm 0,54 \mathrm{a}$ \\
\hline
\end{tabular}

a,b: Les moyennes portant une même lettre sur la même ligne ne sont pas statistiquement différentes $(\mathrm{P}>0,05)$ 
Tableau 3: Effets du charbon de rafles de maïs et de fruits noirs sur le rendement carcasse et le poids relatif de quelques organes $(\%)$ et le taux de la créatinine $(\mathrm{mg} / \mathrm{l})$.

\begin{tabular}{lccc}
\hline & Ro & Rc & Rr \\
\hline Rendement carcasse & $76,91 \pm 2,02 \mathrm{a}$ & $75,36 \pm 0,77 \mathrm{a}$ & $71,96 \pm 3,24 \mathrm{a}$ \\
Foie & $2,50 \pm 0,23 \mathrm{a}$ & $2,68 \pm 0,33 \mathrm{ab}$ & $3,11 \pm 0,21 \mathrm{~b}$ \\
Reins & $1,05 \pm 0,13 \mathrm{a}$ & $1,02 \pm 0,21 \mathrm{a}$ & $1,22 \pm 0,24 \mathrm{a}$ \\
Cœurs & $0,85 \pm 0,13 \mathrm{a}$ & $0,82 \pm 0,24 \mathrm{a}$ & $1,16 \pm 0,15 \mathrm{a}$ \\
Pancréas & $0,32 \pm 0,13 \mathrm{a}$ & $0,44 \pm 0,13 \mathrm{a}$ & $0,51 \pm 0,21 \mathrm{a}$ \\
Graisse abdominale & $1,95 \pm 0,34 \mathrm{a}$ & $1,66 \pm 0,87 \mathrm{a}$ & $1,74 \pm 0,61 \mathrm{a}$ \\
Créatinine (mg/l) & $2,64 \pm 0,12 \mathrm{a}$ & $4,70 \pm 3,02 \mathrm{~b}$ & $2,48 \pm 0,41 \mathrm{a}$ \\
a,b: Les moyennes portant une même lettre sur la même ligne ne sont pas statistiquement différentes (P>0,05)
\end{tabular}

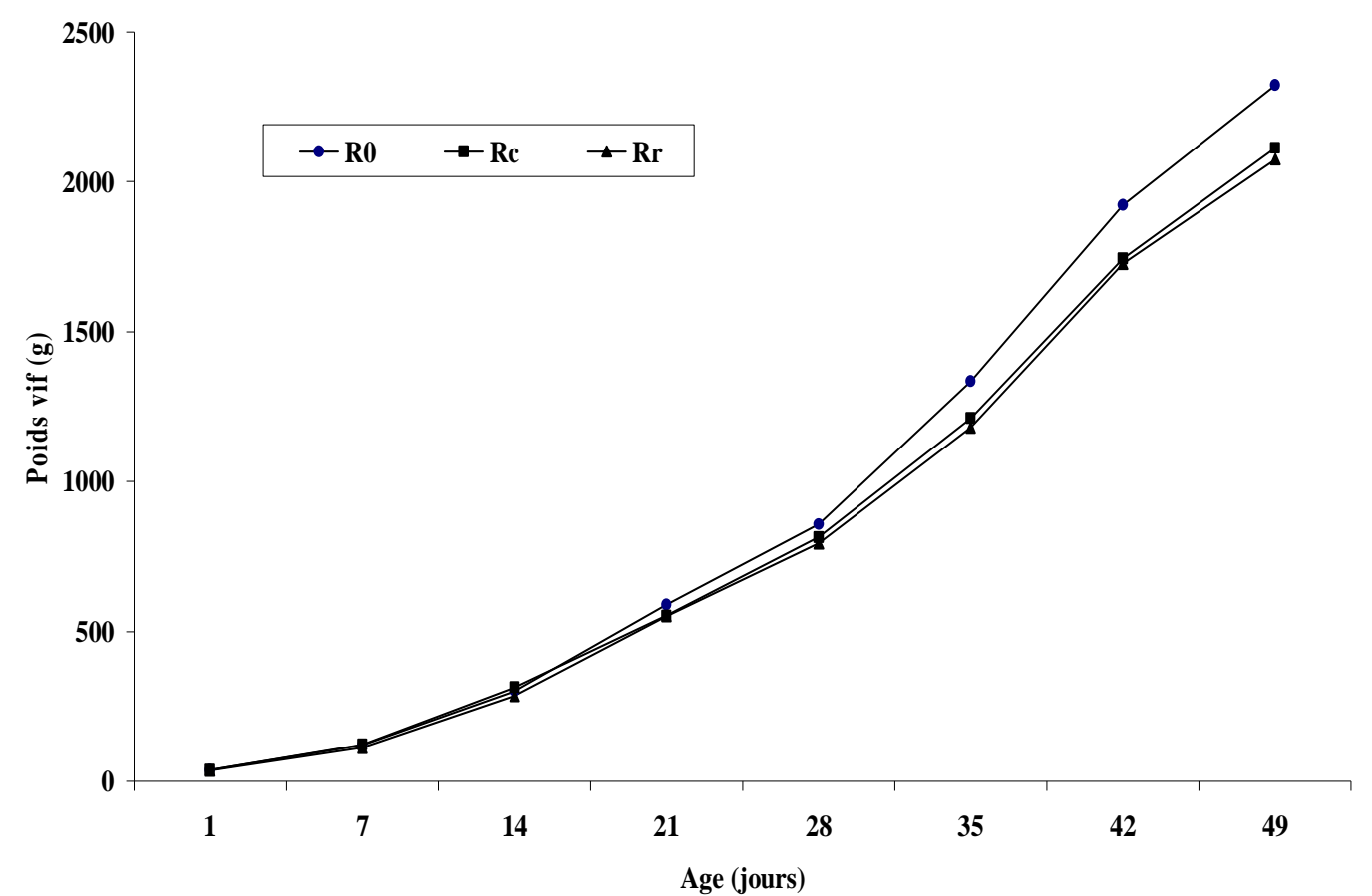

Figure 1: Influence du charbon de fruits noirs $(\mathrm{Rc})$ et de rafles de maïs $(\mathrm{Rr})$ sur les performances pondérales des poulets de chair.

\section{DISCUSSION}

Les résultats de cette étude ont montré que l'incorporation de $1 \%$ du charbon des fruits noirs ou des rafles de maïs dans la ration a des effets dépressifs sur les performances de croissance des poulets de chair. Les charbons sont en général des sources importantes de minéraux qui sont des éléments essentiels pour le développement et la croissance des poulets (Shelton et Southern, 2006). Les 
résultats de cette étude sont contraires aux travaux menés sur la supplémentation des rations des poulets de chair avec des minéraux par Wang et al. (2008). En effet, ces auteurs ont rapporté une nette amélioration des performances de croissance, de la consommation alimentaire et de l'indice de consommation par les minéraux. Ces résultats sont aussi contraires à ceux obtenus avec les feuilles de plante entières (Al-harthi, 2006), les extraits de plante (Gill, 1999; Prakash, 2006; Emadi et Kermanshahi, 2006; Songsak et al., 2008) et les huiles essentielles (OviedoRondon et al., 2006). La baisse des performances enregistrée dans notre étude pourrait être due au taux d'incorporation visiblement très élevé (1\%) du charbon. En effet lorsqu'ils sont incorporés en quantité trop importante dans les rations, la plupart des additifs sont susceptibles d'exercer des effets nocifs sur les animaux (Phillips, 1999).

Les résultats de la présente étude corroborent ceux de Murry et al. (2006) qui n'ont révélé aucun effet bénéfique des probiotiques botaniques sur la croissance pondérale, la consommation alimentaire et l'indice de consommation chez les poulets de chair. D'autres probiotiques comme les levures (Saccharomyces cerevisiae) issues de la fermentation du manioc n'ont eu aucun effet bénéfique sur la consommation alimentaire, le gain de poids et sur les caractéristiques de la carcasse, mais ont eu un effet dépressif sur la morphologie de l'intestin (Songsak et al., 2008).

\section{CONCLUSION}

Dans les conditions de cette étude, les résultats obtenus ont montré que l'incorporation du charbon des fruits noirs et des rafles de maïs a eu un effet dépressif sur les performances de croissance et les caractéristiques de la carcasse chez les poulets de chair, ce qui suggère que le taux d'incorporation du charbon était très élevé. Une variation du taux d'incorporation serait indispensable afin de déterminer le taux optimum d'incorporation et de mieux apprécier le rôle du charbon.

\section{REFERENCES BIBLIOGRAPHIQUES}

Ahmad I. 2006. Effects of probiotics on broilers performance. Int. J. Poult Sc., 5(6): 593-597.
Al-Harthi MA. 2006. Impact of supplemental feed enzyme, condiments mixture or their combination on broiler performance, nutrients digestibility and plasma constituents. Int. J. Poult Sc., 5(8): 764771.

Cooney DO. 1980. Activated Charcoal: Antidotal and other Medical Uses. Marcel Dekker: New York.

Doyle ME. 2002. Alternatives to Antibiotics use for Growth Promotion in Animal Husbandry. FRI Briefings, University of Wisconsin-Madison, WI 53706.

Emadi M, Kermanshahi H. 2006. Effect of tumeric rhizome powder on performance and carcass characteristics of broiler chickens. Int. J. Poult Sc., 5(11): 10691072.

Gunal M, Yayli G, Kaya O, Karahan N, Sulak O. 2006. The effects of antibiotic growth promoter, probiotic or organic acid supplementation on performance, intestinal microflora and tissue of broilers. Int. J. Poult Sc., 5(2): 149-155.

Gill C. 1999. Herbs and plant extracts as growth enhancers. Feed Int., 20(4): 2023.

Jensen BB. 1998. The impact of feed additives on the microbial ecology of the gut in young pigs. J. An Feed Sci., 7: 45-64.

Woo LK, Soo KL, Duk LB. 2006. Aspergillus oryzae as probiotic in poultry - A Review. Int. J. Poult Sc., 5(1): 01-03.

Vilain M. 1999. Méthodes Expérimentales en Agronomie. Pratique et analyse. Editions Tec et Doc.: Paris; 337p.

Murry AC, Hinton A, Buhr RJ. 2006. Effect of botanical probiotic containing Lactobacilli on growth performance ond populations of bacteria in the ceca; cloaca, and carcass rinse of broiler chickens. Int. J. Poult Sc., 5(4): 344-350.

Neuvonen PJ, Olkkola KT. 1988. Oral activated charcoal in the treatment of intoxications. Role of single and repeated doses. Med. Toxicol., 3: 33-58.

Oviedo-Rondon EO, Clemente-Hermandez S, Salvador F, Williams P, Losa R. 2006. Essential oils on mixed coccidian vaccination and infection in broilers. Int. J. Poult Sc., 5(8): 723-730.

Phillips I. 1999. Assessing the evidence that antibiotic growth promoters influence 
human infections. J. Hosp. Infect., 43: 173-178.

Prakash SK. 2006. Effects of herbal extracts towards microbial activity against pathogenic Escherichia coli in poultry. Int. J. Poult Sc., 5(3): 259-261.

Ramos AJ, Johana FG, Hernandez E. 1996. Prevention of toxic effects of mycotoxins by means of non-nutritive absorbent compounds. J. Food Protect., 59(6): 631641.

Shelton JL, Southern LL. 2006. Effects of phytase addition with or without a trace mineral premix on growth performance, bone response variables and tissue mineral concentrations in commercial broilers. J. Apply Poult. Res., 15: 94-102.

Soltan MA. 2008. Effect of dietary organic acid supplementation on egg production, egg quality and some blood serum parameters in laying hens. Int. J. Poult Sc., 7(6): 613-621.

Songsak C, Orawan C, Thassawan S, Sathit N, Sirilak S. 2008. Effect of dietary inclusion of cassava yeast as probiotic source on growth performance, small intestine (Ileum) morphology and carcass characteristic in broilers. Int. J. Poult Sc., 7(3): 246-250.

Wages D. 2002. What does science say about the future of antibiotics? Poult. Int., July 2002. 44 - 46.

Wang Z, Cerrate S, Yan F, Sacakil P, Waldroup PW. 2008. Comparison of different concentrations of inorganic trace minerals in broiler diets on live performance and mineral excretion. Int. J. Poult Sc., 7(7): 625-629. 